\title{
Clinical Applications of Wide-Detector CT Scanners for Cardiothoracic Imaging: An Update
}

\author{
Eun-Ju Kang, MD, PhD \\ Department of Radiology, College of Medicine, Dong-A University, Busan, Korea
}

Technical developments in multidetector computed tomography (CT) have increased the number of detector rows on the z-axis, and $16-\mathrm{cm}$ wide-area-coverage CT scanners have enabled volumetric scanning of the entire heart. Beyond coronary arterial imaging, such innovations offer several advantages during clinical imaging in the cardiothoracic area. The wide-detector CT scanner markedly reduces the image acquisition time to less than 1 second for coronary CT angiography, thereby decreasing the volume of contrast material and radiation dose required for the examination. It also eliminates stair-step artifacts, allowing robust improvements in myocardial function and perfusion imaging. Additionally, new imaging techniques for the cardiothoracic area, including subtraction imaging and free-breathing scans, have been developed and further improved by using the wide-detector CT scanner. This article investigates the technical developments in wide-detector CT scanners, summarizes their clinical applications in the cardiothoracic area, and provides a review of the recent literature.

Keywords: Multidetector computed tomography; Wide-area detector; Coronary artery disease; Imaging; Technology

\section{INTRODUCTION}

Multidetector computed tomography (MDCT) has rapidly developed since the 1980s, and 64-detector CT is an acceptable imaging modality for non-invasive assessment of the coronary arteries. The major difficulties in MDCT for cardiac imaging are the restricted temporal and spatial resolutions and limited $z$-axis coverage, which could produce stair-step artifacts. In this regard, the development of the wide-area-coverage $(16-\mathrm{cm})$ CT scanner has enabled volumetric imaging of the entire heart within one cardiac cycle, free of stair-step artifacts. These advancements have not only improved the diagnostic performance of coronary CT angiography (CCTA) but also lowered the radiation dose

Received May 10, 2019; accepted after revision July 23, 2019. This study was supported by research funds from Dong-A University.

Corresponding author: Eun-Ju Kang, MD, PhD, Department of Radiology, College of Medicine, Dong-A University, 32 Daesingongwon-ro, Seo-gu, Busan 49201, Korea.

- Tel: (8251) 240-5367 - Fax: (8251) 253-4931

- E-mail: medcarrot@dau.ac.kr

This is an 0pen Access article distributed under the terms of the Creative Commons Attribution Non-Commercial License (https://creativecommons.org/licenses/by-nc/4.0) which permits unrestricted non-commercial use, distribution, and reproduction in any medium, provided the original work is properly cited. and contrast material volume. Moreover, they offer several advantages over various clinical imaging modalities, such as triple-rule-out (TRO) scan as well as myocardial perfusion and functional imaging. This article summarizes the technical aspects of commercially available wide-detector CT scanners and suggests their potential clinical applications and benefits in cardiothoracic imaging.

\section{Technical Aspects of the Wide-Detector CT Scanner System}

\section{Development of Wide-Detector MDCT}

Clinical interest in the application of CT for imaging the coronary arteries started in 1998, with the introduction of "4-detector-row" CT scanners. However, these early MDCT models showed limitations in performing coronary angiography; therefore, their use in cardiac applications was confined to coronary calcium scoring, a technique originally established on electron-beam CT scanners (1). Since the development of 16-detector-row CT scanners, CCTA became feasible with the retrospective electrocardiogram (ECG)-gating data-acquisition method; nevertheless, its use is still limited to routine clinical practice (2). Following the introduction of 64-detector-row CT scanners, the diagnostic accuracy of CCTA improved, and it became a 
clinically feasible technique owing to the smaller detector element size $(0.5-0.65 \mathrm{~mm})$ and faster rotation time (350-420 ms). Although 64-detector-row CT represents a marked improvement over conventional MDCT, especially in cardiac imaging, its craniocaudal coverage without gantry movement is typically only $20-40 \mathrm{~mm}$, which limits the width of coverage on cine imaging in the craniocaudal direction.

To overcome this disadvantage, the development of wide-area detectors that would enable $>40-\mathrm{mm}$ coverage per rotation was led by Canon Medical Systems (formerly, Toshiba Medical Systems), Japan. They developed a prototype 256-detector-row CT scanner in 2005, which was designed to allow wide craniocaudal coverage, imaging approximately $100 \mathrm{~mm}$ in one rotation (3-5). Thereafter, the first commercially available wide-range detector scanner was released in November 2007 (Aquilion One; Canon Medical Systems, Otawara, Japan). It had a detector configuration of 320-detector rows $\times 0.5$-mm detector element width, which enabled $16-\mathrm{cm}$ coverage and a $350-\mathrm{ms}$ gantry rotation time at a single focal spot. This configuration allowed threedimensional (3D) volumetric whole-heart imaging during the diastole of one R-R interval; however, the standard temporal resolution was approximately $175 \mathrm{~ms}$ (one-half the gantry rotation time), which remains significantly longer than the $33 \mathrm{~ms}$ achieved using catheter coronary angiography. This 320-detector-row CT scanner shows promising temporal homogeneity and is free of the stair-step artifacts noted when performing volume scans, but it often suffers from cone-beam geometry-related artifacts and insufficient temporal resolution. Since 2011, Canon Medical Systems has adopted the dual focal spot (two alternating z-focal spot positions) technique and a faster gantry rotation time (275 ms). Therefore, the latest-generation wide-detector scanners allow imaging of 640 slices with a $137-\mathrm{ms}$ temporal resolution. GE Healthcare introduced a new volume CT scanner (Revolution CT; GE Healthcare, Waukesha, WI, USA) in 2015; it had 256 detector rows (512 slices), which enabled $16-\mathrm{cm}$ coverage, with a $0.625-\mathrm{mm}$ detector element width, and a gantry rotation speed of $280 \mathrm{~ms}$. In addition, the integration of new algorithms allowed the reduction of cone-beam artifacts $(6,7)$.

Philips Healthcare introduced a wide-coverage scanner in early 2008 (Brilliance iCT; Philips Healthcare, Cleveland, $\mathrm{OH}, \mathrm{USA}$ ), which had 128 detector rows that enabled $8-\mathrm{cm}$ coverage with a $0.625-\mathrm{mm}$ individual detector row width and a 270-ms gantry rotation time. The third-generation dual-source CT scanner SOMATOM Force developed by Siemens Healthineers (Forchheim, Germany) has $6-\mathrm{cm}$ coverage (96-detector rows $\times 0.6$-mm detector element width) but enables fast scan coverage because of the use of double helices and higher pitch values owing to the presence of two X-ray tubes and detector arrays. Generally, the length of the cardiac area that has to be covered in a coronary CT scan is typically around $120-140 \mathrm{~mm}(1)$; therefore, in this review, we will focus on the $16-\mathrm{cm}$ coverage CT scanners (256-320 detector rows) that enable volume scans of the entire heart within one cardiac cycle (Table 1).

\section{Contrast Media and Radiation Dose}

A relatively large proportion of patients suspected to have cardiovascular disease also have accompanying diseases such as diabetes, which is a risk factor for contrast-induced nephropathy (8-10). Moreover, patients with significant stenoses diagnosed on CCTA may require subsequent percutaneous coronary arterial intervention, which will expose them to more amount of contrast material. Therefore, optimization of the contrast material protocol and proper opacification of the vessel lumen are essential in CCTA examinations. Technical improvements in CT systems, with larger numbers of detector rows and faster rotation times, have reduced the image acquisition time, which is also associated with a reduction in the contrast material volume from 100-140 mL for 16-detector-row CT systems to 75-100 mL for 64-detector-row CT systems (11, 12). Wide-detector $\mathrm{CT}$ scanners allow an acquisition time of less than 1 second with single-heartbeat CCTA; therefore,

Table 1. Technical Parameters of Commercially Available Wide-Detector CT Systems

\begin{tabular}{|c|c|c|c|c|c|c|c|c|}
\hline Vendor & CT System & $\begin{array}{l}\text { No. of } \\
\text { Detector } \\
\text { Rows }\end{array}$ & $\begin{array}{l}\text { Detector Element } \\
\text { z-Dimension } \\
(\mathrm{mm})\end{array}$ & $\begin{array}{c}\text { Total Detector } \\
\text { z-Axis Coverage } \\
(\mathrm{mm})\end{array}$ & $\begin{array}{c}\text { Cone } \\
\text { (Degrees) }\end{array}$ & $\begin{array}{l}\text { Rotation } \\
\text { Time } \\
\text { (ms) }\end{array}$ & $\begin{array}{l}\text { Temporal } \\
\text { Resolution } \\
\text { (ms) }\end{array}$ & $\begin{array}{c}\text { X-Ray } \\
\text { Generator } \\
\text { Power (kW) }\end{array}$ \\
\hline \multirow[b]{2}{*}{$\begin{array}{l}\text { Canon Medical } \\
\text { Systems }\end{array}$} & Aquilion ONE & 320 & 0.5 & 160 & 15.2 & 350 & 175 & 72 \\
\hline & $\begin{array}{l}\text { Aquilion ONE vision } \\
\text { Aquilion ONE genesis }\end{array}$ & 320 & 0.5 & 160 & 15.2 & 275 & 137 & 100 \\
\hline GE Healthcare & Revolution CT & 256 & 0.625 & 160 & 10.5 & 280 & 140 & 103 \\
\hline
\end{tabular}

$\mathrm{CT}=$ computed tomography 
these require a very short window of coronary opacification, leading to a marked reduction in the required contrast material volume. Moreover, simultaneous developments in the iterative reconstruction technique in combination with low tube potential (70-100 kV) acquisition have contributed to a reduction in contrast material volume. Several researchers have reported the feasibility of using a lower volume (less than $60 \mathrm{~mL}$ ) of contrast material in wide-detector CT scanners (13-15). In 2011, Kumamaru et al. (13) compared the image quality between the $60-\mathrm{mL}$ and $80-\mathrm{mL}$ contrast material injection protocols for CCTA by using 320-detector-row $\mathrm{CT}$, and they reported that $96 \%$ of the coronary segments showed sufficient enhancement (> 300 Hounsfield units [HU]) when using the $60-\mathrm{mL}$ protocol, thus supporting the general use of $60-\mathrm{mL}$ protocols for clinical wide-area-detector CCTA. Hein et al. (14) reported the diagnostic image quality achieved using $40 \mathrm{~mL}$ of contrast material in prospective ECG-triggered CCTA with a 320-detector-row CT scanner; however, they only enrolled patients with body weight $\leq 85 \mathrm{~kg}$, normal cardiac function, and low heart rate $(\leq 65$ beats per minute $[\mathrm{bpm}])$. Several years later, Kim et al. (15) reported that 320-detector-row CCTA with the 40-mL contrast material protocol showed comparable image quality and diagnostic accuracy to the conventional $60-\mathrm{mL}$ protocol irrespective of patient-related factors; they applied an iterative reconstruction technique (AIDR 3D; Canon Medical Systems), tailored tube voltages $(80,100$, and $120 \mathrm{kVp})$, and tailored contrast injection, which enabled manually-triggered scan commencement by an experienced operator. Nevertheless, optimal enhancement of the coronary arteries for accurate evaluation of coronary arterial stenosis or plaque characterization remains a matter of debate (16-18). Recently published papers proposed that attenuation values $>250-330 \mathrm{HU}$ were acceptable and that values greater than $450 \mathrm{HU}$ may lead to underestimation of coronary arterial stenosis (11-20).

Axial volumetric acquisition of CCTA with a wide-detector CT scanner eliminates redundant radiation exposure from helical oversampling or overlapping of sequential axial acquisition (21); thus, it has the potential to markedly reduce the radiation dose. The initial experience with firstgeneration 320-detector-row CT scanners showed that they yielded a mean effective dose of $8.3 \pm 3.4 \mathrm{mSv}(14.0$ $\mathrm{mSv}$ for retrospective gating and $7.2 \mathrm{mSv}$ for prospective gating), with tube current modulation and modification of the X-ray output based on the patient's body habitus (22). Several years later, the combination of a faster gantry rotation time, iterative reconstruction, and other ancillary developments in CT scanners has provided excellent image quality over a wide range of body sizes and heart rates at low radiation doses (less than $1 \mathrm{mSv}$ ) (23-25).

\section{Effect of Heart Rate and Image Quality}

Heart rate and heart rate variability have been major factors affecting the image quality and difficulties in the interpretation of coronary arteries and may necessitate higher radiation doses in CCTA. In CCTA, there are two types of cardiac motion artifacts that produce poor image quality in the assessment of coronary arteries: in-plane ( $x-y$ axis) motion artifacts that are determined by the temporal resolution (half of the gantry rotation speed) and misregistration artifacts (stair-step artifacts) that are determined by the detector coverage in the $z$-axis $(1,26)$. As mentioned above, the $16-\mathrm{cm}$-coverage widedetector scanners completely avoided misregistration artifacts using volume scans of the heart within one cardiac cycle, particularly in patients with irregular heartbeats. However, in-plane motion artifacts are still a problem in CCTA with wide-detector CT scanners, which depends on the intrinsic gantry rotation time of each CT scanner. Multisegment reconstruction can be used to improve the effective temporal resolution and is available on all kinds of CT scanners using a lower pitch and scanning in the retrospective gated helical scan mode. On the widedetector CT scanner, multisegment reconstruction can also be performed in the prospective ECG-triggered volume mode without full cardiac cycle coverage; however, this technique requires a steady heart rate and generally leads to a higher radiation dose.

Another approach to improving the in-plane motion artifact is the use of vendor-specific motion correction algorithms $(27,28)$. The motion velocity and path of coronary arteries from adjacent cardiac phases are characterized, and the information is used to calculate an optimal estimation of the vessel lumen at the target phase by the snapshot freeze (SSF) reconstruction technique (GE Healthcare) (29). Several previous studies reported that the SSF technique improves diagnostic accuracy using a 64-row detector CT scanner (29-31), and this technique is currently available on wide-detector CT scanners (32-34).

In the 16- $\mathrm{cm}$ wide-detector $\mathrm{CT}$ scanner of GE Healthcare (Revolution CT), using the one-beat auto-gating technique to identify the ideal time within one cardiac cycle at any heart rate in combination with the SSF motion correction 
technique can achieve coronary artery images with diagnostic quality even in patients with a high heart rate or high heart rate variability (32-34).

\section{Applications of Wide-Detector CT in Cardiothoracic Imaging}

Volumetric Cardiac Imaging for Function and Perfusion

Although CCTA is extensively used to evaluate the coronary arteries, it can also provide 3D volumetric information regarding the cardiac chambers or the myocardium. In retrospective ECG-gated CCTA, volumetric left ventricular (LV) functional parameters can be measured simultaneously with the LV mass and volume. The quantification of LV function using CCTA has been validated in many prior studies through a comparison with cardiac MRI, which is considered the gold standard for assessing LV function $(35,36)$. The conventional method for assessing LV function with a 64-detector-row CT scanner is a helical scanning technique with retrospective ECG gating, which covers the entire heart in multiple heartbeats; however, this approach is associated with the risk of motion artifacts due to arrhythmias or breathing (37). The wide-detector CT scanner allows volumetric imaging for a $16-\mathrm{cm} z$-axis coverage with one heartbeat and no time delay; thus, it can acquire both isophasic and isochronic CT data and can yield volumetric and functional measurements of the cardiac chamber with greater accuracy and less radiation exposure $(37,38)$.

Although CT has high sensitivity and negative predictive value for the detection of significant coronary stenosis, the evidence of coronary stenosis is not predictive of inducible ischemia; while the presence and extent of myocardial ischemia are important determinants of adverse outcomes (39-41). Groothuis et al. (42) compared CCTA with MR myocardial perfusion imaging and found that only $42.3 \%$ of patients with obstructive coronary arterial disease on CCTA showed myocardial ischemia on MR perfusion. Thus, additional functional assessments, such as fractional flow reserve (FFR) measurements, cardiac single-photon emission computed tomography (SPECT), stress echocardiography, or stress perfusion $\mathrm{MR}$, are needed to determine the indications for revascularization (42-44). Recent advances in $\mathrm{CT}$ technology have enabled myocardial CT perfusion imaging with high temporal resolution and a reasonable radiation dose. Two approaches in $\mathrm{CT}$ perfusion imagingstatic and dynamic - are currently available. Static CT perfusion imaging refers to the assessment of myocardial enhancement acquired at a single time point of the first pass of the contrast material bolus. Since Kurata et al. (45) first reported static myocardial CT perfusion in 2005, the application of $\mathrm{CT}$ for assessing myocardial ischemia has become widespread, and several clinical studies, including multicenter/multivendor trials, have demonstrated the feasibility of static CT perfusion imaging (46-48). However, the success of static CT perfusion is highly dependent on contrast material bolus timing and cardiac motion, and the single-shot technique cannot provide quantitative values of myocardial perfusion $(49,50)$. In contrast, dynamic CT perfusion is assessed on the basis of myocardial enhancement at multiple time points of the first pass of the contrast material, which is robust in terms of bolus timing and allows for fully quantitative analysis of myocardial perfusion (51). However, the clinical implementation of dynamic CT perfusion assessment has been challenging because of the limited z-axis coverage of 64-detectorrow CT scanners and the relatively high radiation dose inherent to repetitive image acquisition. Although coverage of the LV myocardium is possible using narrower detector scanners in the shuttle mode, the sampling rate for a given part of the myocardium is not high (every second to fourth heartbeat, depending on the heart rate) and the myocardium is temporally different in the cranial and caudal parts $(44,51)$. Wide-detector CT scanners enable dynamic whole-heart volumetric scanning, thereby eliminating the time discrepancy between the base and apex of the heart seen with helical imaging. This opens up the possibility of determining myocardial perfusion precisely (39). In addition, using a wide-detector CT system has several other advantages, including temporal uniformity in image acquisition as well as the possibility of targeting a specific portion of the contrast bolus and reducing the radiation dose and contrast material volume (52). The "CORE-320" multicenter trial group, which was established to support coronary artery evaluation using 320-row MDCT angiography, published several papers regarding the diagnostic accuracy of 320-row MDCT for detecting myocardial perfusion deficits with perfusion CT scanning and compared it with the reference standard of SPECT myocardial perfusion imaging $(48,53-55)$.

\section{Transluminal Attenuation Gradient}

CCTA is an established noninvasive method for anatomic assessment of coronary stenosis; however, it has limited 
specificity for densely calcified lesions and provides no information about their hemodynamic significance. Transluminal attenuation gradient (TAG), defined as the linear regression coefficient between luminal attenuation and axial distance, has been continuously developed since 2010 in an attempt to improve the diagnostic performance of CCTA $(56,57)$. The methodology of TAG is similar in most studies: on the cross-sectional images perpendicular to the centerline for target coronary artery, the mean luminal attenuation is measured at $1-5-\mathrm{mm}$ intervals, from the ostium to the distal level where the lumen area fell below
$2 \mathrm{~mm}^{2}$. Then, TAG is determined from the change in $\mathrm{HU}$ per 10-mm length of coronary artery (Fig. 1). This simple technique retains the functional significance of coronary stenosis without additional radiation exposure or contrast material.

The diagnostic performance of TAG was reported to differ among CT scanners with varied longitudinal coverage, and coronary attenuation variability may occur because of the lack of temporal uniformity among 64-detectorrow CT scanners (58-61). Chow et al. (62) attempted to overcome this inherent limitation of 64-detector-row CT
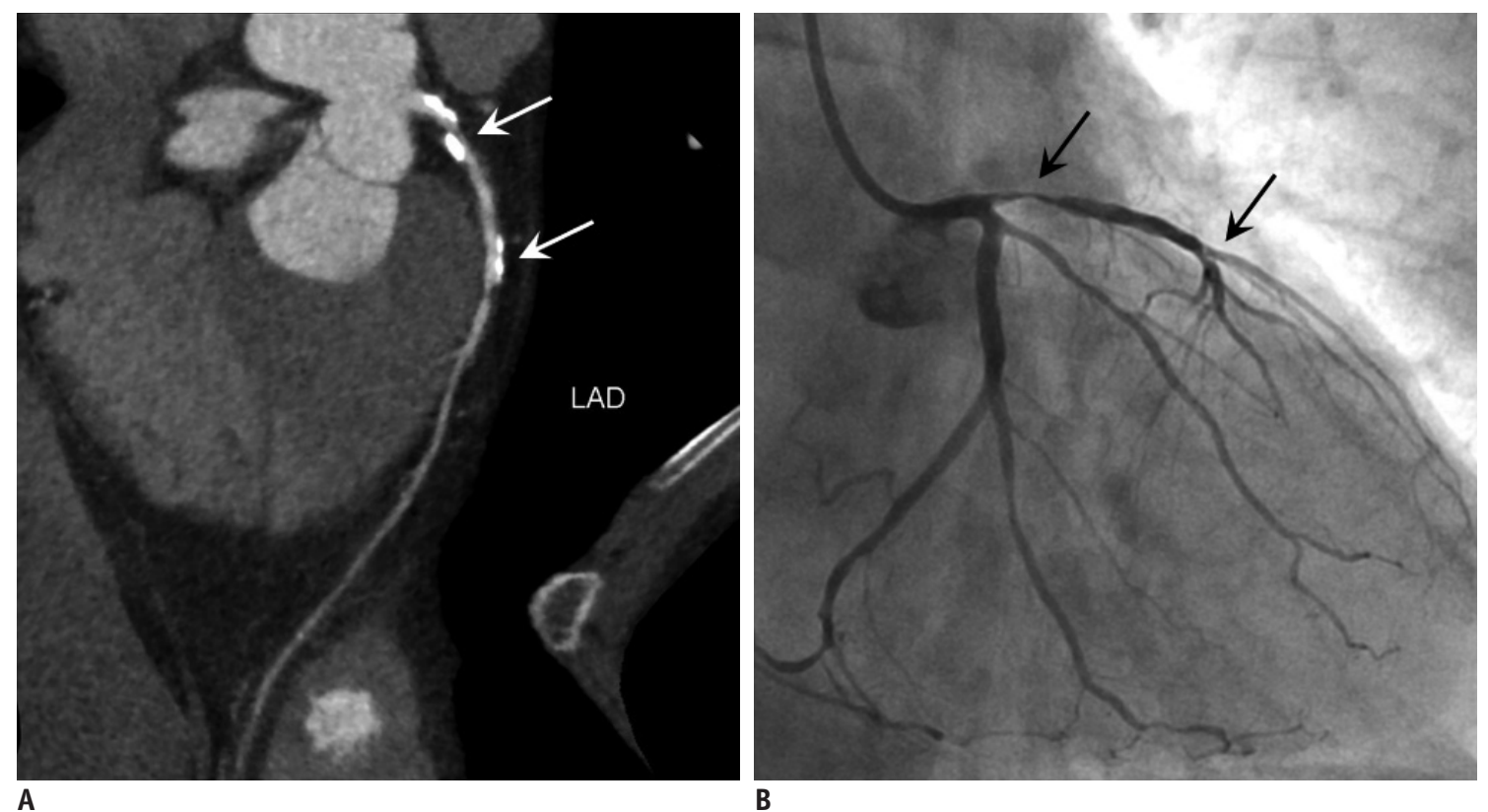

A

B

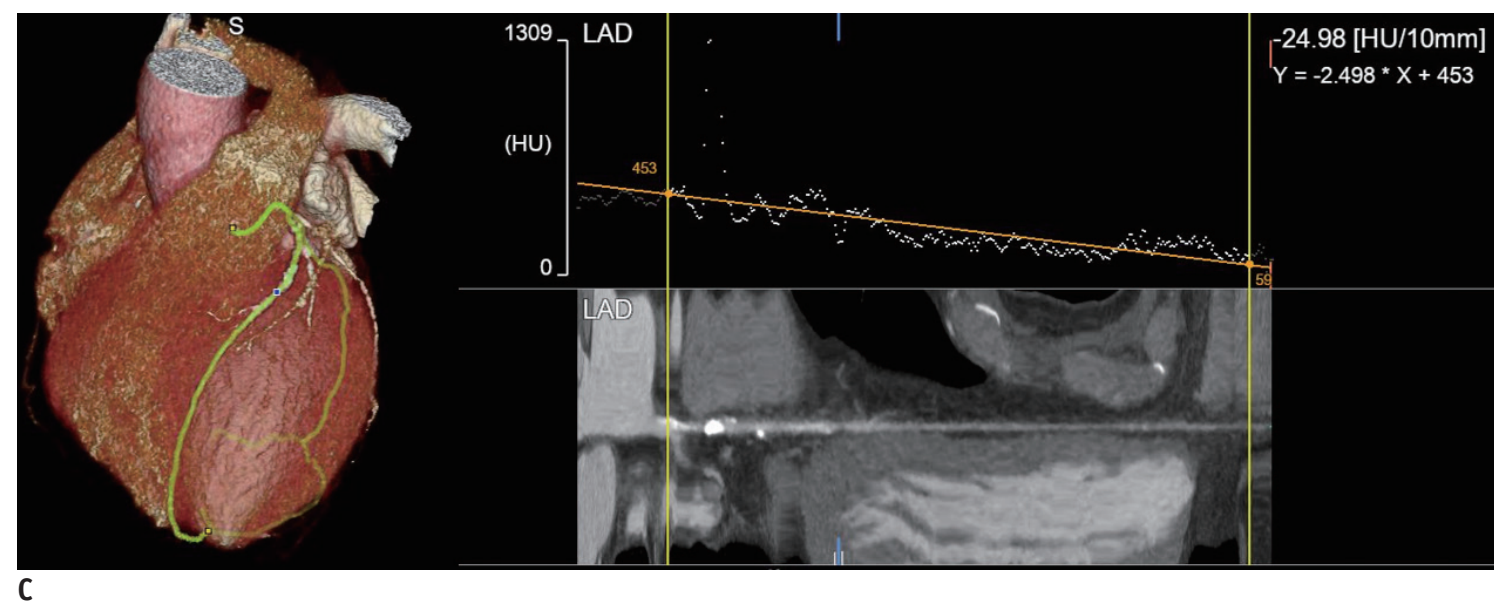

Fig. 1. Representative images of abnormal TAG in LAD of 55-year-old man with nonspecific chest pain.

A. Coronary CT angiography showed LAD with significant dense calcified plaque burden that was suspicious for severe obstructive lesion (arrows). B. Invasive coronary angiography showed severe luminal narrowing of LAD (arrows). C. TAG of LAD measured with semi-automated method by using dedicated computer software (Canon Medical Systems). Mean luminal radiologic attenuation (HU) was measured at 1-mm intervals, from ostium to distal level (cross-sectional area $<2 \mathrm{~mm}^{2}$ ). TAG was -24.98 , which indicates significantly low value (generally accepted cutoff value: -15 to -18). $C T$ = computed tomography, $\mathrm{HU}=$ Hounsfield units, $L A D=$ left anterior descending coronary artery, TAG = transluminal attenuation gradient 
scanners; they suggested corrected coronary opacification (CCO), which was calculated as the quotient of the mean $\mathrm{HU}$ in the coronary artery and aorta $(\mathrm{CCO}=$ coronary artery attenuation/aortic attenuation), to normalize the attenuation values of the coronary lumen to the aorta. Theoretically, whole-heart coverage with single-rotation, single-beat imaging using a wide-detector CT scanner may be ideal for TAG measurement. Several studies have demonstrated that TAG with a 320-detector-row CT scanner has a higher accuracy than does CCTA alone, owing to improvements in specificity and positive predictive value $(59,61)$.

However, clinical validation studies have reported conflicting results despite performing TAG using widedetector CT scanners, and Park et al. (63) suggested that TAG may be affected by changes in the coronary luminal diameter (i.e., the transluminal diameter gradient [TDG]), implying that TAG may be a secondary result of differences in diameters. A recently published study suggested that TAG, TAG-CCO, and TDG do not discriminate between vessels with or without ischemia as defined by quantitative positron emission tomography or FFR (64).

\section{Triple-Rule-Out Scan and Pulmonary Vascular Imaging}

TRO CT protocols can serve as cost-effective diagnostic tools for simultaneously evaluating the coronary arteries, aorta, and pulmonary arteries in patients with acute chest pain; however, these protocols require larger radiation exposures and contrast material volumes to obtain sufficient opacification of the thoracic vessels and appropriate diagnostic image quality. Because of the use of detailedpitch retrospective ECG-gated scanning for the coronary arteries and the overlapping coverage of the same anatomic area for ruling out different causes, TRO CT examinations involve higher radiation doses and larger contrast material volumes owing to the longer scan time compared to that of CCTA. In addition, approximately $10 \%$ of TRO CT images are non-diagnostic in coronary arterial evaluation because of inadequate image quality, especially when using the conventional 64-detector-row CT scanner $(65,66)$.

Various $\mathrm{CT}$ techniques have been proposed to reduce the radiation dose and contrast material volume for TRO $\mathrm{CT}$, including the lower kVp protocol and prospective ECGtriggered high-pitch dual-spiral technique with dual-source CT. However, these techniques could only be applied in specific patient populations: the low $\mathrm{kVp}$ protocol in nonobese patients and the high-pitch mode in patients with a low regular heartbeat (below 65 bpm) (67-69).

Wide-detector CT scanners enable whole-chest CT angiography with two to three axial volume scan acquisitions, which reduce stair-step artifacts and lead to a lower radiation exposure (Fig. 2) (70-72). Kang et al. (72) compared the wide-volume and helical modes of TRO CT using a 320-rowdetector CT scanner and reported that the use of the widevolume scan mode reduces the radiation dose by around $60 \%$ and yields similar image quality to that of helical scans; moreover, even patients with fast or irregular heart rates can be scanned using the wide-volume scan mode and diagnostic images of the coronary arteries can be obtained. Recently, a similar study by Chen et al. (73) using a 256-detector-row CT scanner also reported results consistent with those obtained using a 320-detector-row CT scanner.

\section{Transcatheter Aortic Valve Implantation Evaluation}

Transcatheter aortic valve implantation (TAVI) is a valid alternative therapeutic procedure for patients with symptomatic severe aortic stenosis who are ineligible for surgery or are high-risk surgical candidates $(74,75)$. Currently, the indications for TAVI have expanded to include patients at intermediate risk for conventional surgical valve replacement $(76,77)$. While CT was initially used primarily for the assessment of peripheral access, the role of CT has grown substantially, and it is now the gold standard tool for annular sizing, determining the risk of annular injury and coronary occlusion, and providing appropriate projection angles for fluoroscopic procedures (78). Using a conventional 64- or a 128-row-detector scanner, the TAVI planning CT protocol usually requires two separate acquisitions: a retrospective ECG-gated helical acquisition of the aortic root, followed by an ungated helical acquisition of the aorta and iliofemoral arteries $(78,79)$. This separate acquisition approach requires the injection of two separate boluses of the contrast material, resulting in the administration of a considerable amount of iodine. However, most TAVI candidates are elderly patients who frequently have compromised renal function (80-82). Moreover, accurate measurement of the aortic root annulus maximum size is critical for TAVI planning; thus, image acquisition with retrospective ECG gating to cover the entire cardiac cycle should be considered (78). Therefore, the application of the prospective ultra-high-pitch mode with dual-source scanners seems inappropriate for TAVI planning $(78,82)$.

Many authors have suggested various acquisition protocols for TAVI to reduce the contrast material volume 


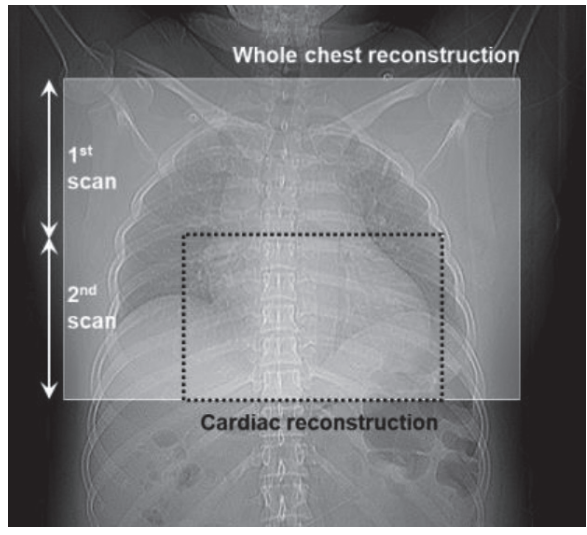

A

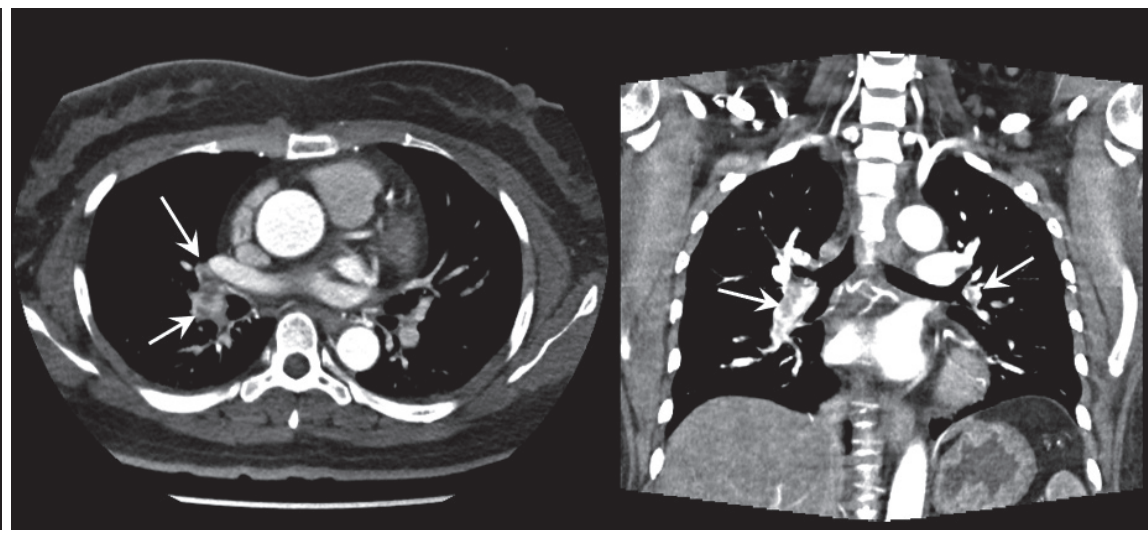

B

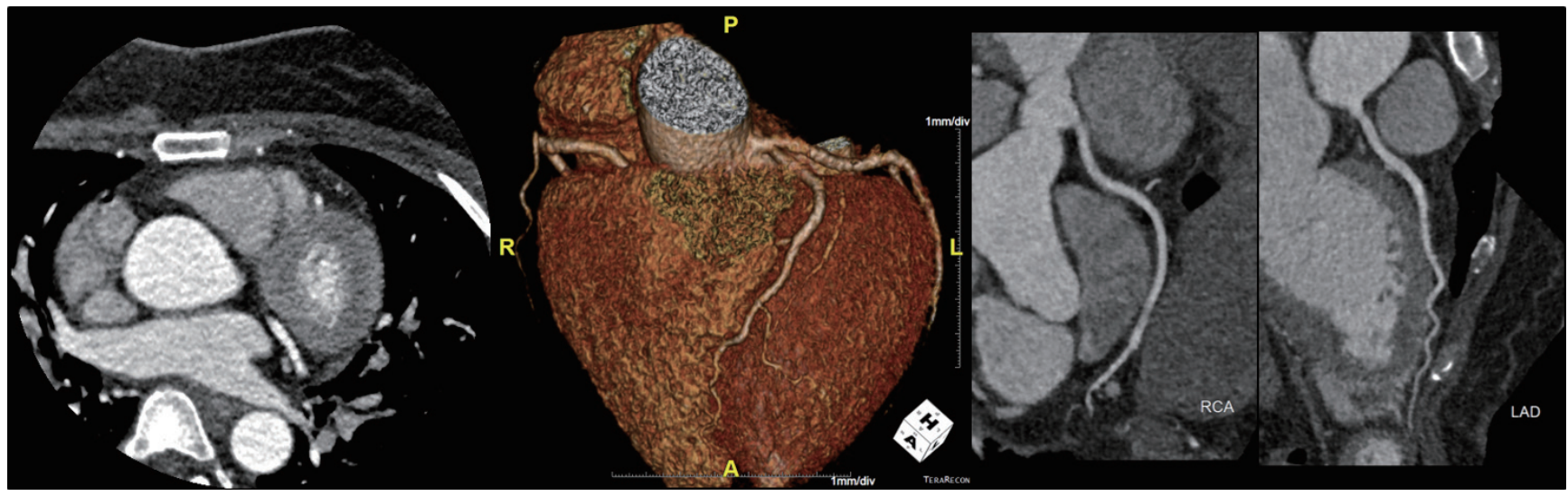

C

Fig. 2. Triple-rule-out scan using wide-volume scan method in 40 -year-old woman diagnosed with acute pulmonary arterial thromboembolism.

A. Detector width is set at $14-16 \mathrm{~cm}$ to cover half of entire thorax, and whole chest is covered with two axial volume scan acquisitions. B. Whole-chest CT scan acquired using two axial volume scans that are automatically stitched immediately after reconstruction. C. Data from second scan are separately reconstructed for coronary angiography. Multifocal low-density filling defects in both lobar and segmental pulmonary arteries suggesting pulmonary arterial thromboembolism (arrows in B). No definite abnormality is observed in thoracic aorta and coronary arteries. RCA = right coronary artery

and radiation dose using the wide-detector CT scanner (7375), and the suggested methods could be roughly divided into two types: 1) axial ECG-gated volume acquisition for the aortic root and heart followed by ungated helical acquisition for the abdomen and pelvis $(82,83)$ and 2$)$ axial ECG-gated volume acquisition for the aortic root and heart followed by three or four ungated volume acquisitions for the abdomen and pelvis (84). According to previous studies, wide-detector CT scanners enable $30-44 \%$ reduction in the iodine load and $32-48 \%$ reduction in the radiation dose in TAVI planning, while maintaining or providing better image quality than that of the conventional protocol using 64-detector-row CT scanners $(82,84)$.

\section{Pediatric Cardiothoracic Imaging}

Wide-detector CT scanners have additional potential advantages in pediatric thoracic imaging beyond their CCTA applications because they can provide rapid imaging of a 16-cm-long anatomic area (which could be the entire thorax in pediatric patients) in a single gantry rotation without table motion (Fig. 3). For pediatric imaging, infant chest CT studies can be performed without sedation using the singlerotation volumetric acquisition mode with a wide-detector CT scanner; this reduces the radiation dose and eliminates the potential risks of sedation (85-87). Zhu et al. (88) compared the image quality, patient preparation time, and radiation dose when performing a single volume scan with 16- $\mathrm{cm}$ wide-detector $\mathrm{CT}$ imaging of the infant chest without sedation to those of 64-detector-row CT imaging with sedation; they found that the scan time was significantly reduced by $83 \%$, preparation time by $57 \%$, and radiation dose (CT dose index) by $42 \%$ when using a wide-detector 

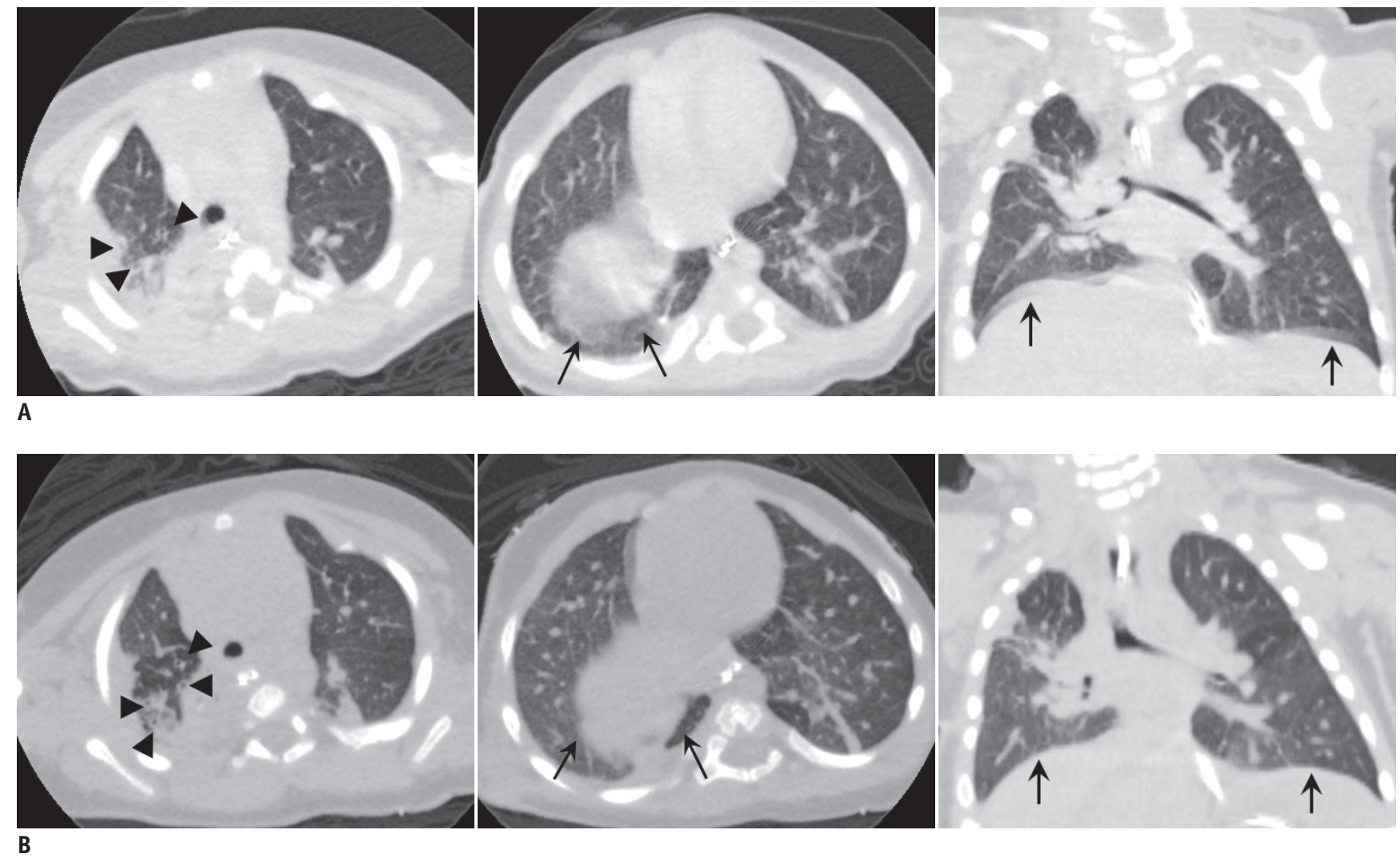

Fig. 3. Thoracic CT images of 4-month-old boy with repaired tracheoesophageal fistula.

Images are obtained using conventional helical acquisition method with sedation (A) and single-axial volume scan method without sedation (B) using 16-cm wide-detector CT scanner. A. Helical mode images show severe motion artifacts with blurring (arrowheads), especially near diaphragm (arrows). B. Axial volume scan images show relatively good image quality with few respiratory motion artifacts near diaphragm (arrows) and more clearly defined lung parenchymal structures, bronchi (arrowheads), vessels, and lung fissures.

CT scanner. The use of wide-detector CT for rapid scanning is best suited for scan lengths less than the 16 -cm width, which limits its application to infants and small children (weighing < $10 \mathrm{~kg}$ ). When the scan length exceeds $16 \mathrm{~cm}$ (in children weighing $>10 \mathrm{~kg}$ ), a delay of at least 2 seconds between the two axial acquisitions increases the overall scan time and can result in greater motion artifacts or a stair-step artifact than when using helical scanning (87-89).

In addition, wide-detector $\mathrm{CT}$ scanners have a potential use in free-breathing dynamic airway evaluation in children suspected of having airway abnormalities such as tracheobronchomalacia (80). Traditionally, airway abnormalities have been diagnosed using bronchoscopy, which has significant disadvantages: it is invasive, requires general anesthesia, and has limited application in infants or small children $(90,91)$. Moreover, stenosis may impede the passage of the bronchoscope and endoscopy may not enable evaluation of the airway distal to a severe stenosis. Therefore, attempts have been made to use MDCT as an alternative evaluation tool for airway assessment (90-
94). However, 64-detector-row MDCT shows an inherent limitation in z-axis coverage, and hence, different airway segments are imaged at varying phases of respiration for acquiring images of the entire central airway (92). The development of a wide-detector CT scanner (with $16 \mathrm{~cm}$ of anatomical coverage) facilitates dynamic imaging of the entire airway at all phases of respiration (90, 95-97). Widedetector CT scanners also allow for volumetric assessment of the airway during free breathing, without anesthesia and without having to move the table through the gantry. Generally, the protocol for dynamic pulmonary scanning involves four to five gantry rotations within a total time of 1.4 seconds and the reconstruction of 8-10 phases (90, $96,97)$. Since the initial study on dynamic airway volume CT in 2010 (the mean effective radiation doses were 5-8.2 $\mathrm{mSv}$ ) (95), researchers have been trying to reduce the radiation dose using low-voltage, low-current, and iterative reconstructions (94-97), and the mean effective dose reported in the most recent study by Andronikou et al. (97) is $1.0 \mathrm{mSv}$. 


\section{Challenging Techniques in Cardiothoracic Imaging:} Free-Breathing Scans and Subtraction Imaging

CCTA examinations are usually performed in a breathholding state to reduce the artifacts caused by respiration. However, CCTA cannot be performed successfully in some patients who cannot hold their breath (e.g., patients who are deaf or have hearing weakness, those with severe pulmonary disease, and those who are uncooperative). Sometimes, the heart rate increases and the variations caused by breath-holding may result in poor image quality. Wide-detector CT scanners are capable of performing one cardiac cycle axial volume scans for CCTA with very short acquisition times without table motion; thus, cardiac shifting caused by respiration during the scan should be insignificant. Kang et al. (98) reported the feasibility of performing CCTA during free breathing by using a firstgeneration 320-detector-row CT scanner in patients with heart rates lower than $75 \mathrm{bpm}$; they also reported that axial volume scans for CCTA resulted in noninferior image quality compared to that acquired using breath-holding CCTA. With the improvements in gantry rotation time and the application of motion-correction algorithms, the latest version of wide-detector CT scanners (256 detector rows) enables the acquisition of images with excellent quality and remarkable diagnostic accuracy despite the high heart rate during the scan $(99,100)$.

Given the improvements in CT technology, CCTA can exclude stenotic coronary arterial lesions with high diagnostic accuracy. Despite these technological advances, which have improved both spatial and temporal resolutions, extensive coronary calcification or stents are still frequently uninterpretable or overestimated in terms of their severity due to blooming artifacts $(101,102)$. Volumetric CCTA acquisition using a wide-detector CT scanner allows coronary subtraction technology, which is a recently developed method that removes calcium and stents from CCTA images. Briefly, the subtraction images are obtained using corresponding non-contrast and contrast datasets, and both of them are registered and subtracted. The result is a $3 \mathrm{D}$ volume in which coronary calcifications/stents have been removed, leaving the contrast-enhanced blood in the lumen as the only high-intensity material $(103,104)$. Since Yoshioka and Tanaka (103) reported the initial experiences with subtraction CCTA using a 320-detector-row CT scanner in 2011, many researchers have attempted to develop better acquisition methods or subtraction algorithms. The most important factors for successful achievement of subtraction CCTA should be accurate registration of the two datasets; therefore, most current studies are based on the single breath-hold method to decrease the likelihood of misregistration between the two datasets (104-108).

The feasibility of subtraction CCTA has been verified by several studies, and subtraction CCTA allows for a significant improvement in diagnostic accuracy owing to its higher specificity and positive predictive value for the assessment of coronary artery stenosis than those of conventional CCTA alone (103-109). However, the proportion of segments excluded because of misregistration is still high even in the most recently published study, which reported misregistration in approximately half (53\%) of all target segments (109). Manual correction can be considered in case of significant lesions, but it is highly time-consuming. Moreover, although the breath-holding times of this single breath-hold method have been shortened to approximately 15-18 seconds, this breath-holding time remains problematic in some patients $(107,108)$. In highly selected patients for whom CCTA can be challenging because of an extensive calcium load or inability to tolerate breathholding, with successful registration, subtraction CCTA may be helpful in the current technique.

\section{CONCLUSION}

Wide-detector CT scanners provide advantages in terms of improvement in image quality and reduction of the radiation dose and contrast material volume in routine CCTA examination than in conventional 64- or 128-detectorrow scanners. Moreover, given their unique characteristic of z-axis coverage, several new technologies (such as myocardial function/perfusion, TAG, or subtraction imaging) have become more feasible and better reflect the exact physiological status of patients. Further development of wide-detector CT scanners, especially for improvements in temporal and spatial resolutions (with faster rotation times and thinner individual detector widths), may provide great clinical advantages in the future.

\section{Conflicts of Interest}

The author has no potential conflicts of interest to disclose.

\section{Acknowledgments}

I would like to offer my special thanks to the staffs of the following organizations for assistance with valuable comments about technology that greatly improved the 
manuscript: Canon Medical Systems Korea, GE Healthcare Korea.

\section{ORCID iD}

Eun-Ju Kang

https://orcid.org/0000-0003-0937-3607

\section{REFERENCES}

1. Lewis MA, Pascoal A, Keevil SF, Lewis CA. Selecting a CT scanner for cardiac imaging: the heart of the matter. $\mathrm{Br} \mathrm{J}$ Radiol 2016;89:20160376

2. Lawler LP, Pannu HK, Fishman EK. MDCT evaluation of the coronary arteries, 2004: how we do it--data acquisition, postprocessing, display, and interpretation. AJR Am J Roentgenol 2005;184:1402-1412

3. Mori S, Endo M, Obata T, Murase K, Fujiwara H, Susumu K, et al. Clinical potentials of the prototype 256-detector row CTscanner. Acad Radiol 2005;12:148-154

4. Mori S, Kondo C, Suzuki N, Yamashita H, Hattori A, Kusakabe $M$, et al. Volumetric cine imaging for cardiovascular circulation using prototype 256-detector row computed tomography scanner (4-dimensional computed tomography): a preliminary study with a porcine model. J Comput Assist Tomogr 2005;29:26-30

5. Mori S, Endo M, Obata T, Tsunoo T, Susumu K, Tanada S. Properties of the prototype 256-row (cone beam) CT scanner. Eur Radiol 2006;16:2100-2108

6. Raju R, Cury RC, Precious B, Blanke P, Naoum C, Arepalli $C$, et al. Comparison of image quality, and diagnostic interpretability of a new volumetric high temporal resolution scanner versus 64-slice MDCT. Clin Imaging 2016;40:205-211

7. Latif MA, Sanchez FW, Sayegh K, Veledar E, Aziz M, Malik R, et al. Volumetric single-beat coronary computed tomography angiography: relationship of image quality, heart rate, and body mass index. Initial patient experience with a new computed tomography scanner. J Comput Assist Tomogr 2016;40:763-772

8. Morcos SK, Thomsen HS, Webb JAW, Contrast Media Safety Committee of the European Society of Urogenital Radiology (ESUR). Contrast-media-induced nephrotoxicity: a consensus report. Eur Radiol 1999;9:1602-1613

9. McDonald RJ, McDonald JS, Bida JP, Carter RE, Fleming $\mathrm{CJ}$, Misra $\mathrm{S}$, et al. Intravenous contrast material-induced nephropathy: causal or coincident phenomenon? Radiology 2013;267:106-118

10. McDonald JS, McDonald RJ, Carter RE, Katzberg RW, Kallmes DF, Williamson EE. Risk of intravenous contrast materialmediated acute kidney injury: a propensity score-matched study stratified by baseline-estimated glomerular filtration rate. Radiology 2014;271:65-73

11. Johnson PT, Pannu HK, Fishman EK. IV contrast infusion for coronary artery CT angiography: literature review and results of a nationwide survey. AJR Am J Roentgenol 2009;192:W214-W221

12. Wintersperger BJ, Nikolaou K. Basics of cardiac MDCT: techniques and contrast application. Eur Radiol 2005;15 Suppl 2:B2-B9

13. Kumamaru KK, Steigner ML, Soga S, Signorelli J, Bedayat A, Adams K, et al. Coronary enhancement for prospective ECGgated single R-R axial 320-MDCT angiography: comparison of 60 - and $80-\mathrm{mL}$ iopamidol 370 injection. AJR Am J Roentgenol 2011;197:844-850

14. Hein PA, May J, Rogalla P, Butler C, Hamm B, Lembcke A. Feasibility of contrast material volume reduction in coronary artery imaging using 320-slice volume CT. Eur Radiol 2010;20:1337-1343

15. Kim R, Park EA, Lee W, Chung JW. Feasibility of 320-row area detector CT coronary angiography using $40 \mathrm{~mL}$ of contrast material: assessment of image quality and diagnostic accuracy. Eur Radiol 2016;26:3802-3810

16. Cademartiri F, Maffei E, Palumbo AA, Malagò R, La Grutta $L$, Meiijboom WB, et al. Influence of intra-coronary enhancement on diagnostic accuracy with 64-slice CT coronary angiography. Eur Radiol 2008;18:576-583

17. Cademartiri F, Mollet NR, Lemos PA, Saia F, Midiri M, de Feyter PJ, et al. Higher intracoronary attenuation improves diagnostic accuracy in MDCT coronary angiography. AJR Am J Roentgenol 2006;187:W430-W433

18. Fei X, Du X, Yang Q, Shen Y, Li P, Liao J, et al. 64-MDCT coronary angiography: phantom study of effects of vascular attenuation on detection of coronary stenosis. AJR Am J Roentgenol 2008;191:43-49

19. Benz DC, Gräni C, Hirt Moch B, Mikulicic F, Vontobel J, Fuchs $\mathrm{TA}$, et al. A low-dose and an ultra-low-dose contrast agent protocol for coronary CT angiography in a clinical setting: quantitative and qualitative comparison to a standard dose protocol. Br J Radiol 2017;90:20160933

20. Tan SK, Yeong CH, Raja Aman RRA, Ng KH, Abdul Aziz YF, Chee $\mathrm{KH}$, et al. Low tube voltage prospectively ECG-triggered coronary CT angiography: a systematic review of image quality and radiation dose. Br J Radiol 2018;91:20170874

21. Hsiao EM, Rybicki FJ, Steigner M. CT coronary angiography: 256-slice and 320-detector row scanners. Curr Cardiol Rep 2010;12:68-75

22. Rybicki FJ, Otero HJ, Steigner ML, Vorobiof G, Nallamshetty $L$, Mitsouras D, et al. Initial evaluation of coronary images from 320-detector row computed tomography. Int J Cardiovasc Imaging 2008;24:535-546

23. Chen MY, Shanbhag SM, Arai AE. Submillisievert median radiation dose for coronary angiography with a secondgeneration 320-detector row CT scanner in 107 consecutive patients. Radiology 2013;267:76-85

24. Benz DC, Gräni C, Mikulicic F, Vontobel J, Fuchs TA, Possner $M$, et al. Adaptive statistical iterative reconstruction-V: impact on image quality in ultralow-dose coronary computed tomography angiography. J Comput Assist Tomogr 
2016;40:958-963

25. Richards CE, Dorman S, John P, Davies A, Evans S, Ninan $\mathrm{T}$, et al. Low-radiation and high image quality coronary computed tomography angiography in "real-world" unselected patients. World J Radiol 2018;10:135-142

26. Kalisz K, Buethe J, Saboo SS, Abbara S, Halliburton S, Rajiah P. Artifacts at cardiac CT: physics and solutions. Radiographics 2016;36:2064-2083

27. Leipsic J, Labounty TM, Hague CJ, Mancini GBJ, O'Brien JM, Wood DA, et al. Effect of a novel vendor-specific motioncorrection algorithm on image quality and diagnostic accuracy in persons undergoing coronary $\mathrm{CT}$ angiography without rate-control medications. J Cardiovasc Comput Tomogr 2012;6:164-171

28. Fan L, Zhang J, Xu D, Dong Z, Li X, Zhang L. CTCA image quality improvement by using snapshot freeze technique under prospective and retrospective electrocardiographic gating. J Comput Assist Tomogr 2015;39:202-206

29. Lee H, Kim JA, Lee JS, Suh J, Paik SH, Park JS. Impact of a vendor-specific motion-correction algorithm on image quality, interpretability, and diagnostic performance of daily routine coronary $\mathrm{CT}$ angiography: influence of heart rate on the effect of motion-correction. Int $\mathrm{J}$ Cardiovasc Imaging 2014;30:1603-1612

30. Carrascosa P, Deviggiano A, Capunay C, De Zan MC, Goldsmit A, Rodriguez-Granillo GA. Effect of intracycle motion correction algorithm on image quality and diagnostic performance of computed tomography coronary angiography in patients with suspected coronary artery disease. Acad Radiol 2015;22:81-86

31. Pontone G, Andreini D, Bertella E, Baggiano A, Mushtaq $S$, Loguercio $M$, et al. Impact of an intra-cycle motion correction algorithm on overall evaluability and diagnostic accuracy of computed tomography coronary angiography. Eur Radiol 2016;26:147-156

32. Liang J, Wang H, Xu L, Dong L, Fan Z, Wang R, et al. Impact of SSF on diagnostic performance of coronary computed tomography angiography within 1 heart beat in patients with high heart rate using a 256-row detector computed tomography. J Comput Assist Tomogr 2018;42:54-61

33. Chen Y, Wei D, Li D, Liu Z, Hu Z, Li M, et al. The value of 16-cm wide-detector computed tomography in coronary computed tomography angiography for patients with high heart rate variability. J Comput Assist Tomogr 2018;42:906911

34. Park JB, Jeong YJ, Lee G, Lee NK, Kim JY, Lee JW. Influence of heart rate and innovative motion-correction algorithm on coronary artery image quality and measurement accuracy using 256-detector row computed tomography scanner: phantom study. Korean J Radiol 2019;20:94-101

35. Raman SV, Shah M, McCarthy B, Garcia A, Ferketich AK. Multi-detector row cardiac computed tomography accurately quantifies right and left ventricular size and function compared with cardiac magnetic resonance. Am Heart J
2006;151:736-744

36. Asferg C, Usinger L, Kristensen TS, Abdulla J. Accuracy of multi-slice computed tomography for measurement of left ventricular ejection fraction compared with cardiac magnetic resonance imaging and two-dimensional transthoracic echocardiography: a systematic review and meta-analysis. Eur J Radiol 2012;81:e757-e762

37. de Graaf FR, Schuijf JD, van Velzen JE, Nucifora G, Kroft LJ, de Roos A, et al. Assessment of global left ventricular function and volumes with 320-row multidetector computed tomography: a comparison with 2D-echocardiography. J Nucl Cardiol 2010;17:225-231

38. Kang EJ, Lee KN, Choi WJ, Kim YD, Shin KM, Lim JK, et al. Left ventricular functional parameters and geometric patterns in Korean adults on coronary CT angiography with a 320-detector-row CT scanner. Korean J Radiol 2017;18:664673

39. Voros S. What are the potential advantages and disadvantages of volumetric CT scanning? J Cardiovasc Comput Tomogr 2009;3:67-70

40. de Roos A. Myocardial perfusion imaging with multidetector CT: beyond lumenography. Radiology 2010;254:321-323

41. Iwasaki K. Myocardial ischemia is a key factor in the management of stable coronary artery disease. World J Cardiol 2014;6:130-139

42. Groothuis JG, Beek AM, Brinckman SL, Meijerink MR, Koestner SC, Nijveldt R, et al. Low to intermediate probability of coronary artery disease: comparison of coronary CT angiography with first-pass MR myocardial perfusion imaging. Radiology 2010;254:384-392

43. Yang DH, Kim YH. CT myocardial perfusion imaging: current status and future perspectives. Int $\mathrm{J}$ Cardiovasc Imaging 2017;33:1009-1020

44. Seitun S, De Lorenzi C, Cademartiri F, Buscaglia A, Travaglio N, Balbi M, et al. CT myocardial perfusion imaging: a new frontier in cardiac imaging. Biomed Res Int 2018;2018:7295460

45. Kurata A, Mochizuki T, Koyama Y, Haraikawa T, Suzuki J, Shigematsu $Y$, et al. Myocardial perfusion imaging using adenosine triphosphate stress multi-slice spiral computed tomography: alternative to stress myocardial perfusion scintigraphy. Circ J 2005;69:550-557

46. Takx RA, Blomberg BA, El Aidi H, Habets J, de Jong PA, Nagel $E$, et al. Diagnostic accuracy of stress myocardial perfusion imaging compared to invasive coronary angiography with fractional flow reserve meta-analysis. Circ Cardiovasc Imaging 2015;8:e002666

47. Cademartiri F, Seitun S, Clemente A, La Grutta L, Toia $P$, Runza $G$, et al. Myocardial blood flow quantification for evaluation of coronary artery disease by computed tomography. Cardiovasc Diagn Ther 2017;7:129-150

48. George RT, Mehra VC, Chen MY, Kitagawa K, Arbab-Zadeh A, Miller JM, et al. Myocardial CT perfusion imaging and SPECT for the diagnosis of coronary artery disease: a head-to- 
head comparison from the CORE320 multicenter diagnostic performance study. Radiology 2014;272:407-416

49. Pelgrim GJ, Nieuwenhuis ER, Duguay TM, van der Geest RJ, Varga-Szemes A, Slump CH, et al. Optimal timing of image acquisition for arterial first pass CT myocardial perfusion imaging. Eur J Radiol 2017;86:227-233

50. van Rosendael AR, de Graaf MA, Dimitriu-Leen AC, van Zwet EW, van den Hoogen IJ, Kharbanda RK, et al. The influence of clinical and acquisition parameters on the interpretability of adenosine stress myocardial computed tomography perfusion. Eur Heart J Cardiovasc Imaging 2017;18:203-211

51. Kitagawa K, Goto Y, Nakamura S, Takafuji M, Hamdy A, Ishida $M$, et al. Dynamic CT perfusion imaging: state of the art. Cardiovasc Imaging Asia 2018;2:38-48

52. George RT, Arbab-Zadeh A, Miller JM, Vavere AL, Bengel FM, Lardo $\mathrm{AC}$, et al. Computed tomography myocardial perfusion imaging with 320-row detector computed tomography accurately detects myocardial ischemia in patients with obstructive coronary artery disease. Circ Cardiovasc Imaging 2012;5:333-340

53. Rochitte CE, George RT, Chen MY, Arbab-Zadeh A, Dewey $M$, Miller JM, et al. Computed tomography angiography and perfusion to assess coronary artery stenosis causing perfusion defects by single photon emission computed tomography: the CORE320 study. Eur Heart J 2014;35:11201130

54. Cury RC, Kitt TM, Feaheny K, Blankstein R, Ghoshhajra BB, Budoff $M J$, et al. A randomized, multicenter, multivendor study of myocardial perfusion imaging with regadenoson CT perfusion vs single photon emission CT. J Cardiovasc Comput Tomogr 2015;9:103-112.e1-e2

55. Chen MY, Rochitte CE, Arbab-Zadeh A, Dewey M, George RT, Miller JM, et al. Prognostic value of combined CT angiography and myocardial perfusion imaging versus invasive coronary angiography and nuclear stress perfusion imaging in the prediction of major adverse cardiovascular events: the CORE320 multicenter study. Radiology 2017;284:55-65

56. Steigner ML, Mitsouras D, Whitmore AG, Otero HJ, Wang C, Buckley 0 , et al. Iodinated contrast opacification gradients in normal coronary arteries imaged with prospectively ECG-gated single heart beat 320-detector row computed tomography. Circ Cardiovasc Imaging 2010;3:179-186

57. Choi JH, Min JK, Labounty TM, Lin FY, Mendoza DD, Shin $\mathrm{DH}$, et al. Intracoronary transluminal attenuation gradient in coronary CT angiography for determining coronary artery stenosis. JACC Cardiovasc Imaging 2011;4:1149-1157

58. Yoon YE, Choi JH, Kim JH, Park KW, Doh JH, Kim YJ, et al. Noninvasive diagnosis of ischemia-causing coronary stenosis using $\mathrm{CT}$ angiography: diagnostic value of transluminal attenuation gradient and fractional flow reserve computed from coronary CT angiography compared to invasively measured fractional flow reserve. JACC Cardiovasc Imaging 2012;5:1088-1096
59. Wong DT, Ko BS, Cameron JD, Nerlekar N, Leung MC, Malaiapan $Y$, et al. Transluminal attenuation gradient in coronary computed tomography angiography is a novel noninvasive approach to the identification of functionally significant coronary artery stenosis: a comparison with fractional flow reserve. J Am Coll Cardiol 2013;61:1271-1279

60. Stuijfzand WJ, Danad I, Raijmakers PG, Marcu CB, Heymans MW, van Kuijk CC, et al. Additional value of transluminal attenuation gradient in CT angiography to predict hemodynamic significance of coronary artery stenosis. JACC Cardiovasc Imaging 2014;7:374-386

61. Ko BS, Wong DT, Nørgaard BL, Leong DP, Cameron JD, Gaur S, et al. Diagnostic performance of transluminal attenuation gradient and noninvasive fractional flow reserve derived from 320-detector row CT angiography to diagnose hemodynamically significant coronary stenosis: an NXT substudy. Radiology 2016;279:75-83

62. Chow BJ, Kass M, Gagné 0, Chen L, Yam Y, Dick A, et al. Can differences in corrected coronary opacification measured with computed tomography predict resting coronary artery flow? J Am Coll Cardiol 2011;57:1280-1288

63. Park EA, Lee W, Park SJ, Kim YK, Hwang HY. Influence of coronary artery diameter on intracoronary transluminal attenuation gradient during CT angiography. JACC CardiovasC Imaging 2016;9:1074-1083

64. Bom MJ, Driessen RS, Stuijfzand WJ, Raijmakers PG, Van Kuijk CC, Lammertsma AA, et al. Diagnostic value of transluminal attenuation gradient for the presence of ischemia as defined by fractional flow reserve and quantitative positron emission tomography. JACC CardiovasC Imaging 2019;12:323-333

65. Goldstein JA, Gallagher MJ, O'Neill WW, Ross MA, O'Neil BJ, Raff GL. A randomized controlled trial of multi-slice coronary computed tomography for evaluation of acute chest pain. $J$ Am Coll Cardiol 2007;49:863-871

66. Hoffmann U, Bamberg F, Chae CU, Nichols JH, Rogers IS, Seneviratne SK, et al. Coronary computed tomography angiography for early triage of patients with acute chest pain: the ROMICAT (rule out myocardial infarction using computer assisted tomography) trial. J Am Coll Cardiol 2009;53:1642-1650

67. Sommer WH, Schenzle JC, Becker CR, Nikolaou K, Graser A, Michalski G, et al. Saving dose in triple-rule-out computed tomography examination using a high-pitch dual spiral technique. Invest Radiol 2010;45:64-71

68. Krissak R, Henzler T, Prechel A, Reichert M, Gruettner J, Sueselbeck T, et al. Triple-rule-out dual-source CT angiography of patients with acute chest pain: dose reduction potential of $100 \mathrm{kV}$ scanning. Eur J Radiol 2012;81:3691-3696

69. Takx RAP, Krissak R, Fink C, Bachmann V, Henzler T, Meyer $M$, et al. Low-tube-voltage selection for triple-rule-out CTA: relation to patient size. Eur Radiol 2017;27:2292-2297

70. Hein PA, Romano VC, Lembcke A, May J, Rogalla P. Initial 
experience with a chest pain protocol using 320-slice volume MDCT. Eur Radiol 2009;19:1148-1155

71. Durmus $T$, Rogalla $P$, Lembcke A, Mühler MR, Hamm B, Hein PA. Low-dose triple-rule-out using 320-row-detector volume MDCT--less contrast medium and lower radiation exposure. Eur Radiol 2011;21:1416-1423

72. Kang EJ, Lee KN, Kim DW, Kim BS, Choi S, Park BH, et al. Triple rule-out acute chest pain evaluation using a 320-rowdetector volume CT: a comparison of the wide-volume and helical modes. Int J Cardiovasc Imaging 2012;28 Suppl 1:713

73. Chen Y, Wang Q, Li J, Jia Y, Yang Q, He T. Triple-rule-out CT angiography using two axial scans with $16 \mathrm{~cm}$ wide-detector for radiation dose reduction. Eur Radiol 2018;28:4654-4661

74. Smith CR, Leon MB, Mack MJ, Miller DC, Moses JW, Svensson LG, et al.; PARTNER Trial Investigators. Transcatheter versus surgical aortic-valve replacement in high-risk patients. $N$ Engl J Med 2011;364:2187-2198

75. Mack MJ, Leon MB, Smith CR, Miller DC, Moses JW, Tuzcu EM, et al.; PARTNER 1 trial investigators. 5-year outcomes of transcatheter aortic valve replacement or surgical aortic valve replacement for high surgical risk patients with aortic stenosis (PARTNER 1): a randomised controlled trial. Lancet 2015;385:2477-2484

76. Leon MB, Smith CR, Mack MJ, Makkar RR, Svensson LG, Kodali SK, et al.; PARTNER 2 Investigators. Transcatheter or surgical aortic-valve replacement in intermediate-risk patients. N Engl J Med 2016;374:1609-1620

77. Reardon MJ, Van Mieghem NM, Popma JJ, Kleiman NS, Søndergaard L, Mumtaz M, et al.; SURTAVI Investigators. Surgical or transcatheter aortic-valve replacement in intermediate-risk patients. N Engl J Med 2017;376:13211331

78. Blanke P, Weir-McCall JR, Achenbach S, Delgado V, Hausleiter $\mathrm{J}$, Jilaihawi $\mathrm{H}$, et al. Computed tomography imaging in the context of transcatheter aortic valve implantation (TAVI) / transcatheter aortic valve replacement (TAVR): an expert consensus document of the Society of Cardiovascular Computed Tomography. $J$ Cardiovasc Comput Tomogr 2019;13:1-20

79. Achenbach S, Delgado V, Hausleiter J, Schoenhagen P, Min JK, Leipsic JA. SCCT expert consensus document on computed tomography imaging before transcatheter aortic valve implantation (TAVI)/transcatheter aortic valve replacement (TAVR). J Cardiovasc Comput Tomogr 2012;6:366-380

80. Gilard M, Eltchaninoff $H$, Iung B, Donzeau-Gouge $P$, Chevreul $\mathrm{K}$, Fajadet J, et al.; FRANCE 2 Investigators. Registry of transcatheter aortic-valve implantation in high-risk patients. N Engl J Med 2012;366:1705-1715

81. Zahn R, Gerckens U, Grube E, Linke A, Sievert H, Eggebrecht $\mathrm{H}$, et al.; German Transcatheter Aortic Valve InterventionsRegistry Investigators. Transcatheter aortic valve implantation: first results from a multi-centre real-world registry. Eur Heart J 2011;32:198-204

82. Mata-Mbemba D, Labani A, El Ghannudi S, Jeung MY, Ohlmann P, Roy C, et al. 320-row CT transcatheter aortic valve replacement planning with a single reduced contrast media bolus injection. PLoS One 2018;13:e0204145

83. Pulerwitz TC, Khalique OK, Nazif TN, Rozenshtein A, Pearson GD, Hahn RT, et al. Very low intravenous contrast volume protocol for computed tomography angiography providing comprehensive cardiac and vascular assessment prior to transcatheter aortic valve replacement in patients with chronic kidney disease. J Cardiovasc Comput Tomogr 2016;10:316-321

84. Shuman WP, Green DE, Busey JM, Ramos MM, Branch KR, Koprowicz KM, et al. Wide-detector axial CT versus $4 \mathrm{~cm}$ detector helical $\mathrm{CT}$ for transcatheter aortic valve replacement: iodine dose, radiation, and image quality. Clin Imaging 2016;40:1213-1218

85. Kroft LJ, Roelofs JJ, Geleijns J. Scan time and patient dose for thoracic imaging in neonates and small children using axial volumetric 320-detector row CT compared to helical 64-, 32-, and 16- detector row CT acquisitions. Pediatr Radiol 2010;40:294-300

86. Podberesky DJ, Angel E, Yoshizumi TT, Toncheva G, Salisbury SR, Brody AS, et al. Comparison of radiation dose estimates and scan performance in pediatric high-resolution thoracic CT for volumetric 320-detector row, helical 64-detector row, and noncontiguous axial scan acquisitions. Acad Radiol 2013;20:1152-1161

87. Ryu YJ, Kim WS, Choi YH, Cheon JE, Lee SM, Cho HH, et al. Pediatric chest CT: wide-volume and helical scan modes in 320-MDCT. AJR Am J Roentgenol 2015;205:1315-1321

88. Zhu Y, Li Z, Ma J, Hong Y, Pi Z, Qu X, et al. Imaging the infant chest without sedation: feasibility of using single axial rotation with $16-\mathrm{cm}$ wide-detector CT. Radiology 2018;286:279-285

89. Gottumukkala RV, Kalra MK, Tabari A, Otrakji A, Gee MS. Advanced CT techniques for decreasing radiation dose, reducing sedation requirements, and optimizing image quality in children. Radiographics 2019;39:709-726

90. Greenberg SB. Dynamic pulmonary CT of children. AJR Am J Roentgenol 2012;199:435-440

91. Mok Q, Negus S, McLaren CA, Rajka T, Elliott MJ, Roebuck $D J$, et al. Computed tomography versus bronchography in the diagnosis and management of tracheobronchomalacia in ventilator dependent infants. Arch Dis Child Fetal Neonatal Ed 2005;90:F290-F293

92. Baroni RH, Feller-Kopman D, Nishino M, Hatabu H, Loring $\mathrm{SH}$, Ernst A, et al. Tracheobronchomalacia: comparison between end-expiratory and dynamic expiratory CT for evaluation of central airway collapse. Radiology 2005;235:635-641

93. Lee EY, Boiselle PM. Tracheobronchomalacia in infants and children: multidetector CT evaluation. Radiology 2009;252:722 
94. Lee KS, Sun MRM, Ernst A, Feller-Kopman D, Majid A, Boiselle PM. Comparison of dynamic expiratory CT with bronchoscopy for diagnosing airway malacia: a pilot evaluation. Chest 2007;131:758-764

95. Wagnetz U, Roberts HC, Chung T, Patsios D, Chapman KR, Paul NS. Dynamic airway evaluation with volume CT: initial experience. Can Assoc Radiol J 2010;61:90-97

96. Greenberg SB, Dyamenahalli U. Dynamic pulmonary computed tomography angiography: a new standard for evaluation of combined airway and vascular abnormalities in infants. Int J Cardiovasc Imaging 2014;30:407-414

97. Andronikou S, Chopra M, Langton-Hewer S, Maier P, Green $\mathrm{J}$, Norbury E, et al. Technique, pitfalls, quality, radiation dose and findings of dynamic 4-dimensional computed tomography for airway imaging in infants and children. Pediatr Radiol 2019;49:678-686

98. Kang EJ, Lee J, Lee KN, Kown H, Ha DH, Kim RB. An initial randomised study assessing free-breathing CCTA using 320-detector CT. Eur Radiol 2013;23:1199-1209

99. Liu Z, Sun Y, Zhang Z, Chen L, Hong N. Feasibility of freebreathing CCTA using 256-MDCT. Medicine (Baltimore) 2016;95:e4096

100. Liu Z, Zhang Z, Hong N, Chen L, Cao C, Liu J, et al. Diagnostic performance of free-breathing coronary computed tomography angiography without heart rate control using 16-cm z-coverage CT with motion-correction algorithm. J Cardiovasc Comput Tomogr 2019;13:113-117

101. Park MJ, Jung JI, Choi YS, Ann SH, Youn HJ, Jeon GN, et al. Coronary $\mathrm{CT}$ angiography in patients with high calcium score: evaluation of plaque characteristics and diagnostic accuracy. Int J Cardiovasc Imaging 2011;27 Suppl 1:43-51

102. Andrew M, John $\mathrm{H}$. The challenge of coronary calcium on coronary computed tomographic angiography (CCTA) scans: effect on interpretation and possible solutions. Int J Cardiovasc Imaging 2015;31 Suppl 2:145-157

103. Yoshioka K, Tanaka R. Subtraction coronary CT angiography for the evaluation of severely calcified lesions using a 320-detector row scanner. Curr Cardiovasc Imaging Rep 2011;4:437-446

104. Tanaka R, Yoshioka K, Muranaka K, Chiba T, Ueda T, Sasaki T, et al. Improved evaluation of calcified segments on coronary CT angiography: a feasibility study of coronary calcium subtraction. Int J Cardiovasc Imaging 2013;29 Suppl 2:75-81

105. Fuchs A, Kühl JT, Chen MY, Helqvist S, Razeto M, Arakita $\mathrm{K}$, et al. Feasibility of coronary calcium and stent image subtraction using 320-detector row CT angiography. J Cardiovasc Comput Tomogr 2015;9:393-398

106. Viladés Medel D, Leta R, Alomar Serralach X, Carreras Costa $F$, Pons-Lladó G. Reliability of a new method for coronary artery calcium or metal subtraction by 320 -row cardiac CT. Eur Radiol 2016;26:3208-3214

107. Kidoh M, Utsunomiya D, Oda S, Yuki H, Funama Y, Namimoto $\mathrm{T}$, et al. Optimized subtraction coronary CT angiography protocol for clinical use with short breath-holding timeinitial experience. Acad Radiol 2015;22:117-120

108. Yamaguchi T, Ichikawa K, Takahashi D, Sugaya T, Furuya $\mathrm{J}$, Igarashi K. A new contrast enhancement protocol for subtraction coronary computed tomography requiring a short breath-holding time. Acad Radiol 2017;24:38-44

109. Fuchs A, Kühl JT, Chen MY, Viladés Medel D, Alomar X, Shanbhag SM, et al. Subtraction CT angiography improves evaluation of significant coronary artery disease in patients with severe calcifications or stents-the C-sub 320 multicenter trial. Eur Radiol 2018;28:4077-4085 